\title{
Machine Learning Vs Deep Learning: Which Is Better For Human Activity Recognition
}

\author{
Nasim Uddin, Mayank Pratap Singh, M. Vaidhehi Rakesh
}

\begin{abstract}
Human activity recognition(HAR) is used to describe basic activities that humans are performing using the sensors that we have in smartphones. The data for this activity recognition is captured by various sensors of mobile phones or wristbands such as accelerometer, gyroscope and gravity sensors.HAR has grabbed the attention of various researchers due to its vast demand in the fields of sport training, security, entertainment health monitoring,computer vision and robotics. In this project we compare different machine learning and deep learning algorithms to find a better approach for HAR. The dataset comprises six activities i.e. walking, sleeping, sitting,moving upward, moving downwards and standing.In this demonstration we also showed confusion matrix,accuracy and multi log loss of various algorithms. With the help of accuracy, confusion matrix of algorithms we compare and determine the best approach for HAR. This will help in future research to map the activities of humans using one of the best approaches used.

Keywords: Human activity recognition, machine learning, deep learning.
\end{abstract}

\section{INTRODUCTION}

Human activity recognition (HAR) is an interesting topic in the research areas. People around the world use smartphones. These smartphones have various sensors, accelerometer and gyroscope sensors are one of them. Accelerometer sensor measures the gravitational acceleration of the device on which it is installed in.To detect the movement in multidirectional an accelerometer is designed with a multi axis sensor. Gyroscope sensors are used to measure the angular velocity of the object. The data obtained from these sensors are put into machine learning algorithms to recognise the events happening there.hence it is used for various practical applications in real time environment such as observing patients for health diagnosis, it can also be applied for automated surveillance to predict crimes. It also can be used in robotics to predict human activity. There are two ways in which human activities are recognised i.e. using wearables and external. Using wearables we can recognise the basic activities of human such as standing,sitting,going upstairs,going downstairs,laying down and walking but using external we can the detailed information like eating, shower,washing dishes,cooking etc.this is due to the fact these sensors

Revised Manuscript Received on April 25, 2020.

* Correspondence Author

Nasim Uddin*, Department of Computer Science and Engineering SRM Institute of Science and Technology Chennai,India MayankPratapSingh, Department of Computer Science and Engineerin, SRM Institute of Science and Technology Chennai,India

Mrs. M. Vaidhehi Rakesh, Department of Computer Science and Engineering

TechnologyChennai, India SRM Institute of Science and

(C) The Authors. Published by Blue Eyes Intelligence Engineering and Sciences Publication (BEIESP). This is an open access article under the CC BY-NC-ND license (http://creativecommons.org/licenses/by-nc-nd/4.0/) capture the data from the device that humans interact with such as faucets, washing machine,stove etc. But these sensors have high installation and maintenance cost.Cameras can also be used as a sensor to gather the information for HAR. It can gather the activities of humans from the video sequences. These activity monitoring can be used in security applications. The thoughts of human activity recognition has been carried from the very past according to the literature. People have used applying using several methods.there is always a discussion on the choice of machine learning and deep learning. People focus more on deep learning approach so as to get better outcome.deep learning provides a higher level of abstraction to the data which results in robust models. Normally in a deep learning approach the output of the previous layer is taken and transform them so as to form a new output.Deep learning is used in most fields including voice recognition,computer vision,natural language processing and image processing. Uses of deep learning in these areas have the proof that it performs better than the standard methods of machine learning. In this paper we will compare deep learning approach to standard machine learning methods.

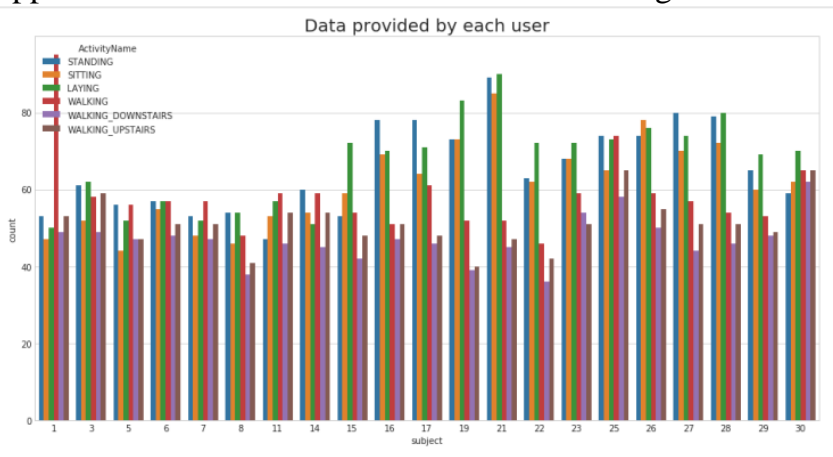

\section{RELATED WORKS}

In [1] Mehdi Hassan, Md. zia uddin and Amr Mohamed used smartphones sensors and deep learning approach for HAR. It contains three phase sensing,feature extraction and recognition. For modeling facial expressions they used pretraining and pre-tuning through deep brief network. Nils Hammerla, Shane holloran and Thomas plotz [2] used wearables data to recognize the human activity. They used daphnet gait, PAMAP, opportunity dataset to understand their work and precise output. Charissa Ann Ranao, SungBae Cho [3] used deep learning neural network for activity recognition. All the complex activities such as bathing ,washing dishes are composed of basic activities such as standing,sitting, laying down. It used deep convolutional neural network for efficiency.It provide automatic data adaptability and robust features without preprocessing. Daniel Ravi,

Published By:

Blue Eyes Intelligence Engineering DOI: 10.35940/ijeat.C6310.049420

Journal Website: www.ijeat.org 1344 \& Sciences Publication

(C) Copyright: All rights reserved.

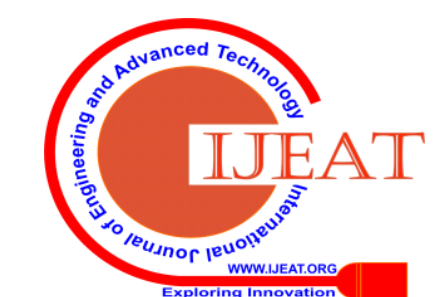




\section{Machine Learning Vs Deep Learning: Which Is Better For Human Activity Recognition}

Charence Wong, Benny LO and Guang-Zhong Yang used deep learning for low powered devices[4]. Henry Friday Nweke, Ying Wah Teh, Mohammed Ali Al-garadi,Uzoma Rita Alo[5] used various deep learning algorithms for activity recognition. They used sparse coding,convolutional neural network, Recurrent neural networks,Restricted Boltzman machine,deep autoencoder. In [6] binary space time map calculation is used in deep learning algorithm for human activity recognition.They did this with two steps which has five process human detection,posture extraction,shadows extraction binary space time map calculation and deep learning recognition.Some paper [7] used convolutional neural network for activity recognition.Hristijan Gjoreski, Jani Bizjak,Martin Gjoreski, Matjaz Gams[8] used various deep learning and machine learning algorithms on data obtained from wrist accelerometer and compared them to find the better approach.

Tushar Dobhal, Vivswan Shitole,Gabriel thomas and Girisha Navada[9] proposed a deep learning method which uses binary motion image.to subtract binary backgrounds used to create binary motion image, the authors used Gaussian Mixture Models.

In [10]authors proposed an activity recognition technique which used depth images.three derived images are extracted from this depth images. The three derived images are Average depth image,motion history image and depth difference image.they used deep belief network using restricted Boltzmann Machine.

In[11] andrej K. et. al. proposed a convolutional neural network approach,they used two ConvNet channels,one channel is fed to a low resolution image and the other is fed to high resolution image.then these two channels converge into two fully converted layers.

Gracia-Ceja et al. [12] presented a paper for complex activities such as shopping,dinner and exercise using wrist band.

In [13]a discrete fourier transformation was applied to the image and the output is used for classification. In $[14,15,16]$ data is taken from the accelerometer of a device the authors mapped the $\mathrm{x}$ - $\mathrm{y}$ - and $\mathrm{z}$ - channels of accelerometer separately and then activity recognition is done.

\section{PROPOSED SYSTEM}

\section{A. Linear regression with grid search}

Linear Regression basically refers to a linear way of determining the connection(or relationship) among the dependent(scalar) and independent variables(one or more),the former being called as simple linear regression and the latter being referred to as multiple linear regression.The task of a linear regression algorithm is to determine the dependent variable value(y2) based upon the independent variable(x2).

Hypothesis function for the above technique is given by:$\mathrm{y} 2=\mathrm{A}+\mathrm{B} \cdot \mathrm{x} 2$, where $\mathrm{A}$ refers to the intercept and $\mathrm{B}$ refers to the coefficient of $\mathrm{x} 2$.Now,in order to get the best fit line for the model,one way is to start with random values of A and B and then accordingly changing the values of $\mathrm{A}$ and $\mathrm{B}$ till we get the minimum estimated cost value and the other way could be by determining minimum cost function i.e minimum RMSE(Root Mean Squared Error) value between y2 and the estimated value.

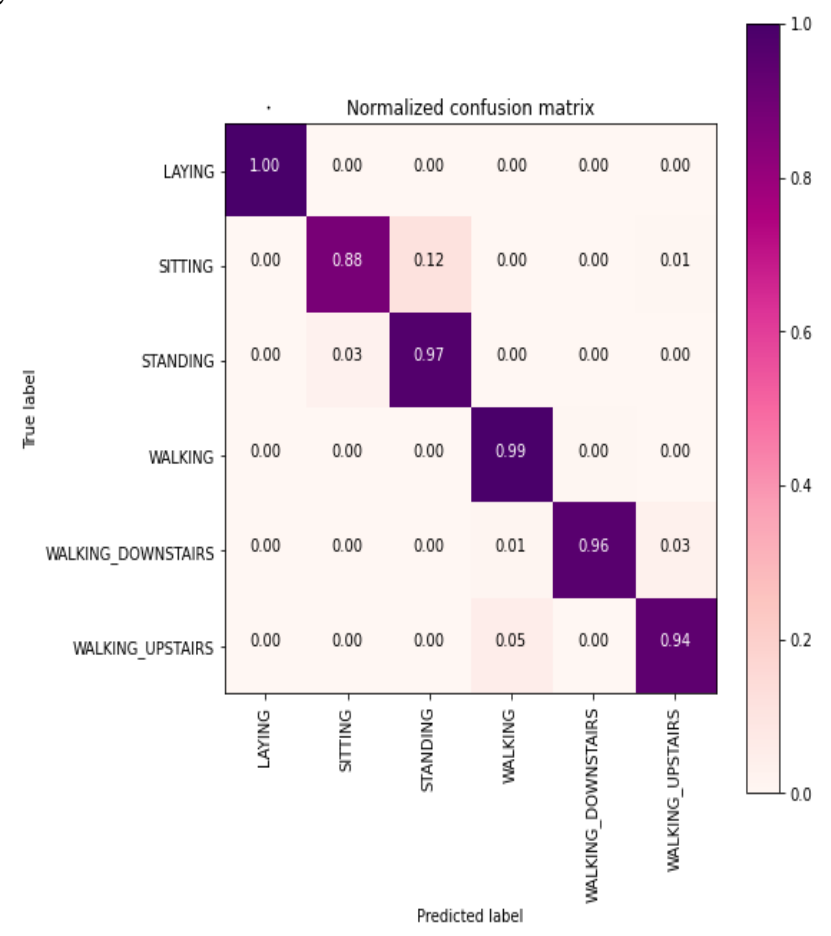

Classification Report

\begin{tabular}{|l|l|l|l|l|}
\hline & Precision & Recall & fl-score & support \\
\hline LAYING & 1.00 & 1.00 & 1.00 & 537 \\
\hline SITTING & 0.97 & 0.87 & 0.92 & 491 \\
\hline STANDING & 0.90 & 0.98 & 0.94 & 532 \\
\hline WALKING & 0.95 & 1.00 & 0.97 & 496 \\
\hline $\begin{array}{l}\text { WALKING } \\
\text { UPSTAIRS }\end{array}$ & 1.00 & 0.97 & 0.99 & 420 \\
\hline $\begin{array}{l}\text { WALKING } \\
\text { DOWNSTAIRS }\end{array}$ & 0.97 & 0.95 & 0.96 & 471 \\
\hline avg/total & 0.96 & 0.96 & 0.96 & 2947 \\
\hline
\end{tabular}

\section{B. Decision tree with grid search}

This is basically the most common and most efficient tool used for classification and prediction.This basically refers to a hierarchical type flowchart where each node generally refers to the test being performed on the given attribute,the branch determines the result of that test,and the terminal node refers to the final label or the final result of the total process of classification. Decision trees help in classifying the instances by sorting them from the starting (root) node of the tree all the way down to the leaf node,which is the required result of the given problem.In this we generally take the general conditions for the particular problem,and then start splitting them into different possible ways,in 
which each splitting then becomes the node(or situation) which can be further split(sometimes not).Thus this process is continued till we reach a condition where further splitting does not give any result,or splitting is not possible.

Classification Report

\begin{tabular}{|l|l|l|l|l|}
\hline & Precision & Recall & fl-score & support \\
\hline LAYING & 1.00 & 1.00 & 1.00 & 537 \\
\hline SITTING & 0.81 & 0.79 & 0.80 & 491 \\
\hline STANDING & 0.81 & 0.83 & 0.82 & 532 \\
\hline WALKING & 0.84 & 0.95 & 0.89 & 496 \\
\hline $\begin{array}{l}\text { WALKING } \\
\text { UPSTAIRS }\end{array}$ & 0.88 & 0.82 & 0.85 & 420 \\
\hline $\begin{array}{l}\text { WALKING } \\
\text { DOWNSTAIRS }\end{array}$ & 0.84 & 0.78 & 0.81 & 471 \\
\hline avg/total & 0.86 & 0.86 & 0.86 & 2947 \\
\hline
\end{tabular}

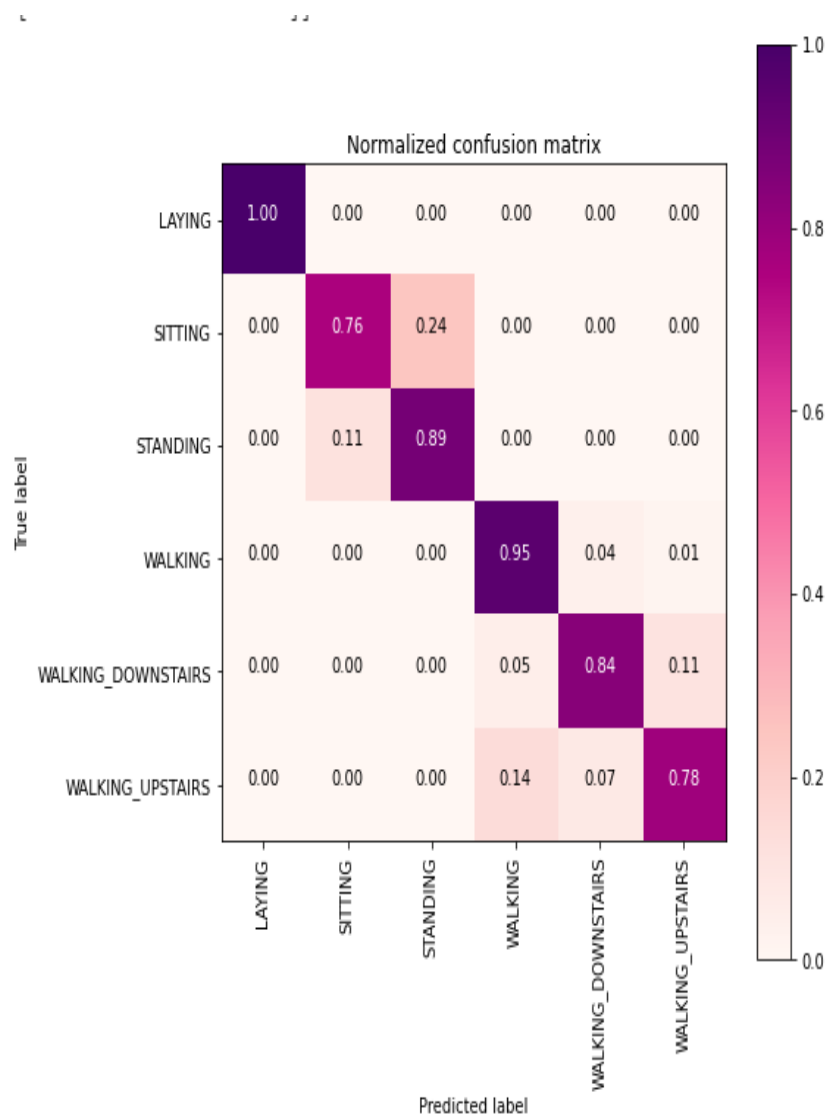

\section{Linear SVC with grid search}

Support Vector Classifier is being developed with an objective to fit to the data which is being provided,thereby returning the hyperplane that divides,categorises the data in the best or most accurate way.Thereby after getting the hyperplane we can provide some new data to the classifier to determine the predicted class. Certain advantages of linear svc are,different regularisation in the formulation can be applied.It is relatively faster and more accurate than the non linear classifier.It is a convex optimisation problem i.e can achieve global optimum,for which other methods such as SMO are available.An approximation to a bound on test error rate is there,and there is a substantial theory behind this which suggests it should be a good idea. Classification Report

\begin{tabular}{|l|l|l|l|l|}
\hline & Precision & Recall & f1-score & support \\
\hline LAYING & 1.00 & 1.00 & 1.00 & 537 \\
\hline SITTING & 0.97 & 0.87 & 0.92 & 491 \\
\hline STANDING & 0.90 & 0.97 & 0.94 & 532 \\
\hline WALKING & 0.97 & 1.00 & 0.99 & 496 \\
\hline $\begin{array}{l}\text { WALKING } \\
\text { UPSTAIRS }\end{array}$ & 1.00 & 0.98 & 0.99 & 420 \\
\hline $\begin{array}{l}\text { WALKING } \\
\text { DOWNSTAIRS }\end{array}$ & 0.98 & 0.97 & 0.97 & 471 \\
\hline avg total & 0.97 & 0.97 & 0.97 & 2947 \\
\hline
\end{tabular}

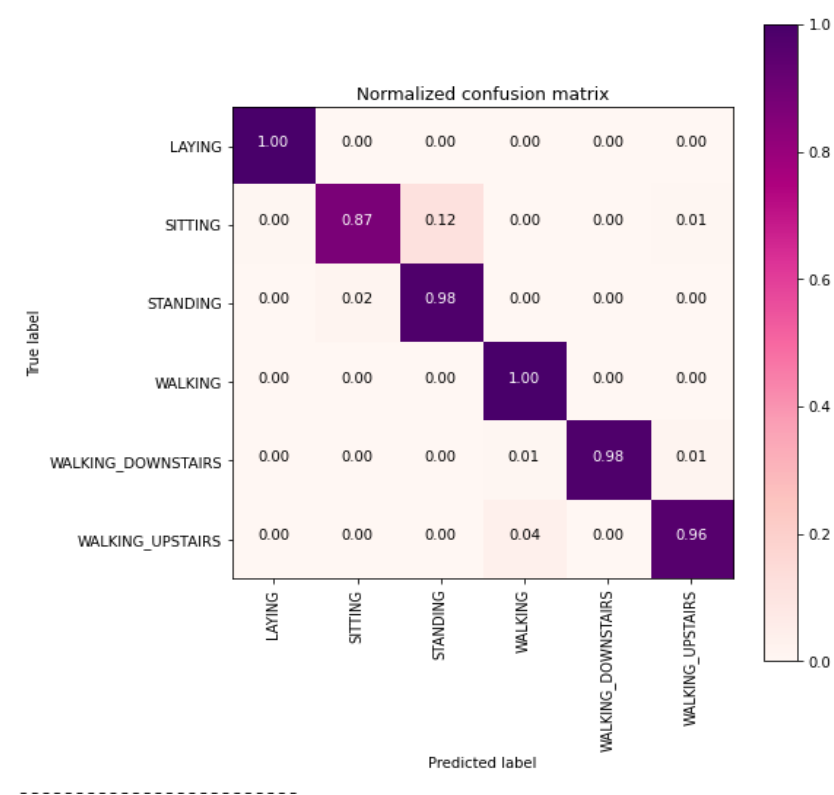

\section{Random forest classifier with grid search}

Random forest is a kind of ensemble classifier which uses decision tree algorithm in a randomized way.It develops a large number of decision trees based on random selection of data and the variables within that data.It provides the class of dependent variables based upon the results obtained from various trees.This algorithm generates a large number of trees based upon random selection of data generally termed as Bootstrap Data.This leads to the creation of random forest.This method is useful in the sense that suppose we want to determine the value of a target variable given a tuple.So we feed that tuple to various randomly generated trees,and find out or store the results being generated.Then there is process of voting being done i.e.the results from different trees are being counted and the result which gets

Published By:

Blue Eyes Intelligence Engineering

\& Sciences Publication

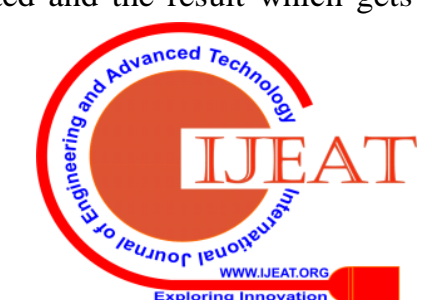


the large number of votes is our required answer for the target value.

Classification Report

\begin{tabular}{|l|l|l|l|l|}
\hline & Precision & Recall & f1-score & support \\
\hline LAYING & 1.00 & 1.00 & 1.00 & 537 \\
\hline SITTING & 0.89 & 0.87 & 0.88 & 491 \\
\hline STANDING & 0.88 & 0.90 & 0.89 & 532 \\
\hline WALKING & 0.87 & 0.98 & 0.92 & 496 \\
\hline $\begin{array}{l}\text { WALKING } \\
\text { UPSTAIRS }\end{array}$ & 0.95 & 0.79 & 0.86 & 420 \\
\hline $\begin{array}{l}\text { WALKING } \\
\text { DOWNSTAIRS }\end{array}$ & 0.89 & 0.92 & 0.90 & 471 \\
\hline avg/total & 0.92 & 0.91 & 0.91 & $k 947$ \\
\hline
\end{tabular}

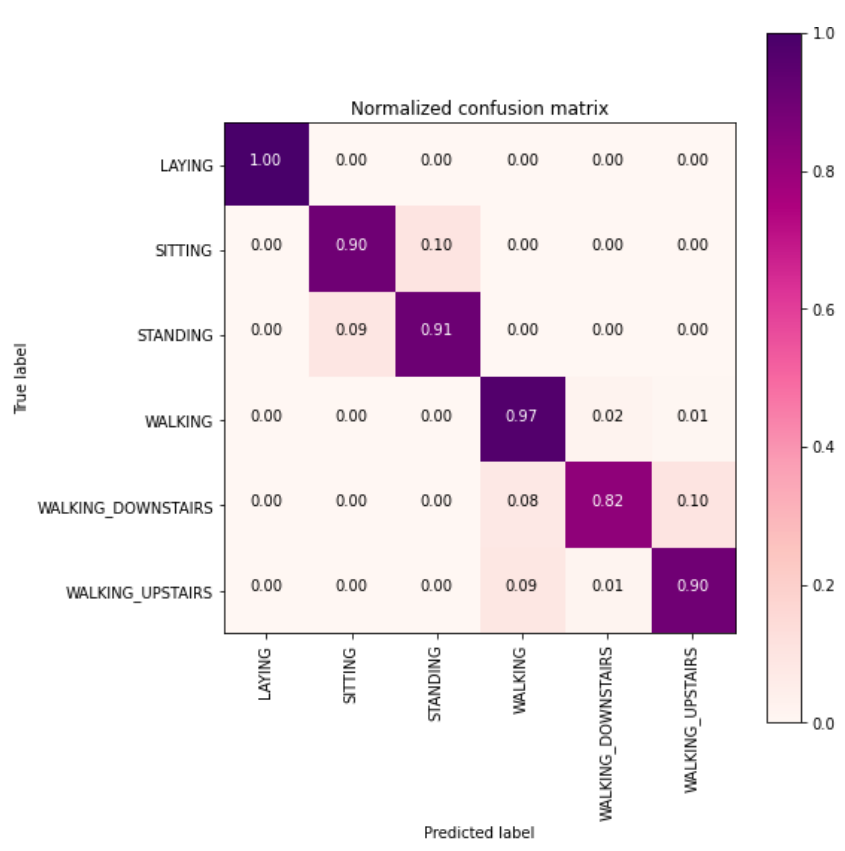

This method is useful in the sense that suppose we want to determine the value of a target variable given a tuple.So we feed that tuple to various randomly generated trees,and find out or store the results being generated.Then there is process of voting being done i.e.the results from different trees are being counted and the result which gets the large number of votes is our required answer for the target value.

\section{E. Gradient boosted classifier with grid search:}

This is a Machine Learning algorithm which is basically a type of ensemble learning.In this technique we generally build up the model in series i.e here we build up the models which help in reducing errors sequentially.For e.g:-suppose we have built a model which produces some kind of errors,therefore now as we build up our second model we keep one thing in mind that the newly developed model will reduce the errors being produced by the first model,similarly we build up our third,fourth and so on.In other words, it is trying to develop new sequential model for hard to fit data.Gradient boosting basically trains model in a gradual,additive and sequential manner.Gradient boosting identifies the shortcomings of weak learner models by using gradients in the loss function which is a measure indicating how good the model's coefficient are at fitting the underlying data.

$\mathrm{V}=\mathrm{Ax}+\mathrm{B}+\mathrm{C}$, where $\mathrm{C}$ refers to the error term.

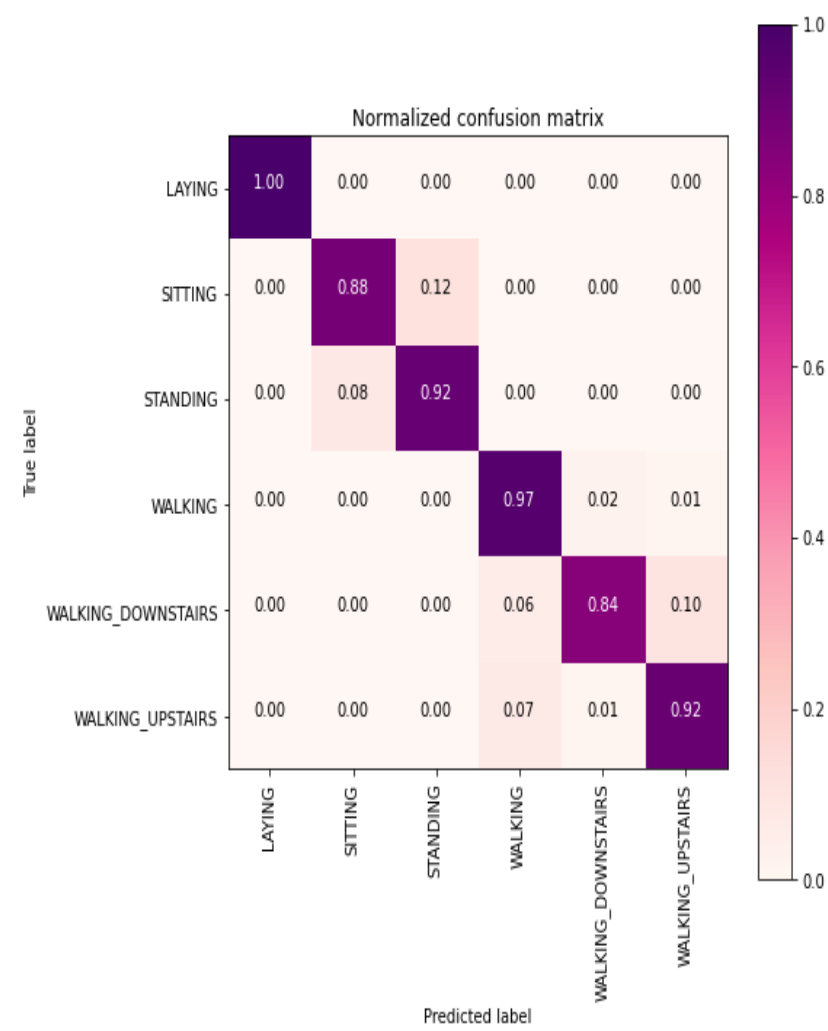

Classification Report

\begin{tabular}{|l|l|l|l|l|}
\hline & Precision & Recall & f1-score & support \\
\hline LAYING & 1.00 & 1.00 & 1.00 & 537 \\
\hline SITTING & 0.91 & 0.81 & 0.86 & 491 \\
\hline STANDING & 0.84 & 0.93 & 0.88 & 532 \\
\hline WALKING & 0.92 & 0.97 & 0.95 & 496 \\
\hline $\begin{array}{l}\text { WALKING } \\
\text { UPSTAIRS }\end{array}$ & 0.97 & 0.89 & 0.93 & 420 \\
\hline $\begin{array}{l}\text { WALKING } \\
\text { DOWNSTAIRS }\end{array}$ & 0.91 & 0.92 & 0.91 & 471 \\
\hline avg total & 0.92 & 0.92 & 0.92 & 2947 \\
\hline
\end{tabular}

\section{F. Kernel SVM using grid search}

SVM algorithms are generally using a set of mathematical functions generally referred to as the Kernel.Its basic function is to consume certain type of data as input and convert it into the desired form.Different types of kernel functions like linear,non-linear,polynomial,radial basis function,sigmoid etc. are being used by different types of SVM algorithms.The most Common type of kernel function is the radial basis function,since it has localised and finite response along the entire $\mathrm{x}$-axis. 
This function generally returns the inner product between the two points in a suitable feature space,thereby defining a notion of equivalence with low computational cost in highdimensional spaces.

Classification Report

\begin{tabular}{|l|l|l|l|l|}
\hline & Precision & Recall & f1-score & support \\
\hline LAYING & 1.00 & 1.00 & 1.00 & 537 \\
\hline SITTING & 0.97 & 0.90 & 0.93 & 491 \\
\hline STANDING & 0.92 & 0.98 & 0.95 & 532 \\
\hline WALKING & 0.96 & 0.99 & 0.97 & 496 \\
\hline $\begin{array}{l}\text { WALKING } \\
\text { UPSTAIRS }\end{array}$ & 0.99 & 0.95 & 0.97 & 420 \\
\hline $\begin{array}{l}\text { WALKING } \\
\text { DOWNSTAIRS }\end{array}$ & 0.95 & 0.96 & 0.95 & 471 \\
\hline avg/total & 0.96 & 0.96 & 0.96 & 2947 \\
\hline
\end{tabular}

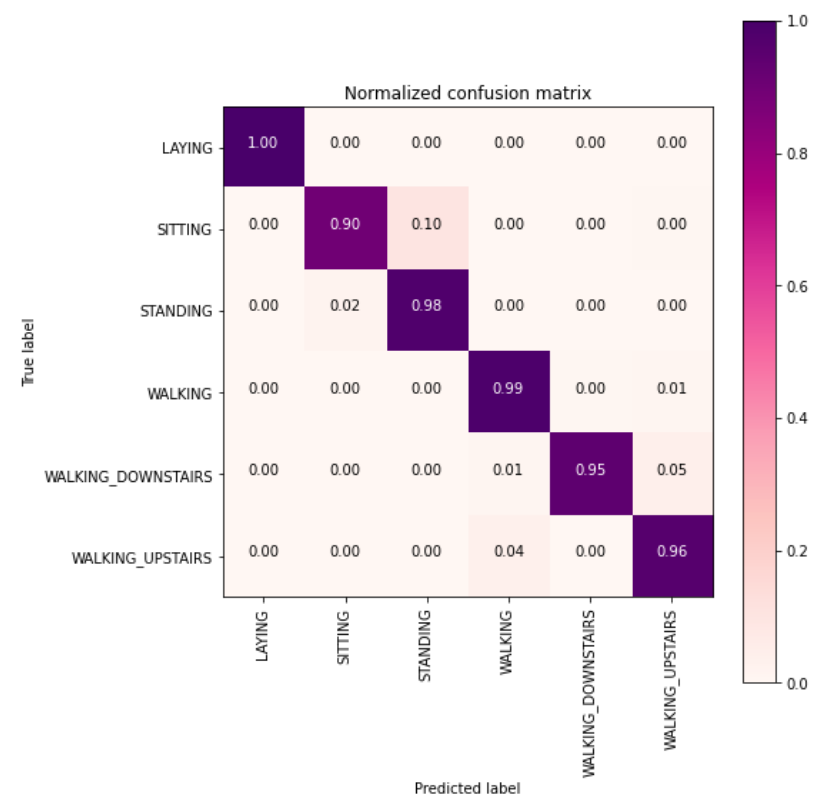

\section{G. LSTM}

It is an artificial Recurrent Neural network most commonly used in the field of deep learning.LSTM is generally a feedback neural network which means it can not only process single data points(like a image) but also the entire stream of data(video or audio).it is also applicable on unsegmented,speech recognition and anomaly detection in network traffic.A common LSTM is generally made up of a cell,an input gate,output gate and a forget gate.

The cell retains the values over arbitrary time intervals and the present three gates regulate or control the flow of information in and out of the cell.

LSTM are best suited for classification,processing and making predictions on the basis of time series data,since there can be several lags of miscellaneous durations between important events.They are built up to deal with exploding and vanishing gradient problems that can be encountered when training traditional RNN.Relative insensitivity to gap length is an advantage of LSTM over the other RNN.

\section{RESULT}

We got the dataset from University of California,Irvine. Where they got the data from 30 individuals by obtaining their data from smartphones. These are actually the data of the accelerometer and gyroscope meter. We used these data in machine learning models as well as deep learning models. But there are two forms of data. One is expert engineered data and the other is raw data. We used expert engineered data in the machine learning algorithms and raw data in the deep learning algorithm. From our result we got to know that several machine learning algorithms got the accuracy above $90 \%$. And the accuracy of logistic regression, linear SVC, and kernel SVM has accuracy above $96 \%$. Which is very good. We used the raw data in the deep learning model and got the accuracy of $91 \%$ which is far good because we used raw data. And also due to less data the deep learning algorithm got the accuracy of $91 \%$. If we had more data we would have got a good accuracy as compared to machine learning algorithms.

\begin{tabular}{|l|l|l|}
\hline & Accuracy(\%) & Error(\%) \\
\hline Logistic Regression & 96.3 & 3.699 \\
\hline Linear SVC & 96.5 & 3.495 \\
\hline Kerne/ SVM & 96.27 & 3.733 \\
\hline Decision Tree & 86.39 & 13.61 \\
\hline Random Forest Classifier & 91.08 & 8.924 \\
\hline $\begin{array}{l}\text { Gradient Boosted Decision } \\
\text { Trees }\end{array}$ & 91.08 & 8.924 \\
\hline
\end{tabular}

\section{CONCLUSION}

We used 561 expert engineer features and applied machine learning algorithms to them. For every model we made confusion matrix.we saw from the confusion matrix that we have a confusion in sitting and standing position.in linear regression we saw that in true label it is $87 \%$ of sitting probability and predicted that $12 \%$ of sitting position as standing position.which changes the accuracy of the model.and the same we saw in other models too.while comparing we observed that the accuracy of linear regression,linear SVC and kernel svm are nearly 96\%.but the decision tree,random forest and gradient booster have decent accuracy.By applying a single LSTM model with no signal processing we got accuracy of about $91 \%$. As we have less time series data we were able to get a decent accuracy in deep learning model. If we use a larger time series data we can make complex LSTM models whose result will be comparable to machine learning models plus the expert features.this can further be improved if we use hyperparameter tuning with larger dropout rates and also by applying more than one LSTM layer. Accuracy can also be improved by increasing LSTM units.so here we conclude that human activity recognition can be improved using deep learning models.

\section{REFERENCES}

1. Hassan, M.M., Uddin, M.Z., Mohamed, A. and Almogren, A., 2018. A robust human activity recognition system using smartphone sensors and deep learning. Future Generation Computer Systems, 81, pp.307313.

Published By:

Blue Eyes Intelligence Engineering

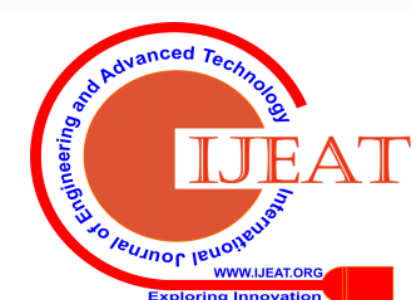


2. Hammerla, N.Y., Halloran, S. and Plötz, T., 2016. Deep, convolutional, and recurrent models for human activity recognition using wearables. arXiv preprint arXiv:1604.08880.

3. Ronao, C.A. and Cho, S.B., 2016. Human activity recognition with smartphone sensors using deep learning neural networks. Expert systems with applications, 59, pp.235-244.

4. Ravi, D., Wong, C., Lo, B. and Yang, G.Z., 2016, June. Deep learning for human activity recognition: A resource efficient implementation on low-power devices. In 2016 IEEE 13th international conference on wearable and implantable body sensor networks (BSN) (pp. 71-76). IEEE.

5. Nweke, H.F., Teh, Y.W., Al-Garadi, M.A. and Alo, U.R., 2018. Deep learning algorithms for human activity recognition using mobile and wearable sensor networks: State of the art and research challenges. Expert Systems with Applications, 105, pp.233-261.

6. Khelalef, A., Ababsa, F. and Benoudjit, N., 2019. An Efficient Human Activity Recognition Technique Based on Deep Learning. Pattern Recognition and Image Analysis, 29(4), pp.702-715.

7. Zebin, T., Scully, P.J. and Ozanyan, K.B., 2016, October. Human activity recognition with inertial sensors using a deep learning approach. In 2016 IEEE SENSORS (pp. 1-3). IEEE.

8. Gjoreski, H., Bizjak, J., Gjoreski, M. and Gams, M., 2016, July. Comparing deep and classical machine learning methods for human activity recognition using wrist accelerometer. In Proceedings of the IJCAI 2016 Workshop on Deep Learning for Artificial Intelligence, New York, NY, USA (Vol. 10).

9. Dobhal, T., Shitole, V., Thomas, G. and Navada, G., 2015. Human activity recognition using binary motion image and deep learning. Procedia computer science, 58, pp.178-185.

10. Foggia, P., Saggese, A., Strisciuglio, N. and Vento, M., 2014, August. Exploiting the deep learning paradigm for recognizing human actions. In 2014 11th IEEE International Conference on Advanced Video and Signal Based Surveillance (AVSS) (pp. 93-98). IEEE.

11. Karpathy, A., Toderici, G., Shetty, S., Leung, T., Sukthankar, R. and Fei-Fei, L., 2014. Large-scale video classification with convolutional neural networks. In Proceedings of the IEEE conference on Computer Vision and Pattern Recognition (pp. 1725-1732).

12. Garcia-Ceja, E., Brena, R., Carrasco-Jimenez, J. and Garrido, L., 2014. Long-term activity recognition from wristwatch accelerometer data. Sensors, 14(12), pp.22500-22524.

13. Jiang, W. and Yin, Z., 2015, October. Human activity recognition using wearable sensors by deep convolutional neural networks. In Proceedings of the 23rd ACM international conference on Multimedia (pp. 1307-1310). Acm.

14. Zeng, M., Nguyen, L.T., Yu, B., Mengshoel, O.J., Zhu, J., Wu, P. and Zhang, J., 2014, November. Convolutional neural networks for human activity recognition using mobile sensors. In 6th International Conference on Mobile Computing, Applications and Services (pp. 197205). IEEE.

15. Ronao, C.A. and Cho, S.B., 2016. Human activity recognition with smartphone sensors using deep learning neural networks. Expert systems with applications, 59, pp.235-244.

16. Ronao, C.A. and Cho, S.B., 2015, November. Deep convolutional neural networks for human activity recognition with smartphone sensors. In International Conference on Neural Information Processing (pp. 46-53). Springer, Cham.

\section{AUTHORS PROFILE}

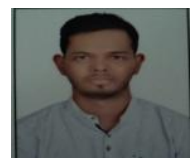

Nasim Uddin, C.S.E , B.TECH

SRM Institute of Science and Technology,

Chennai,Email:- nasim20nk@gmail.com

Mob.no.:-8668163565

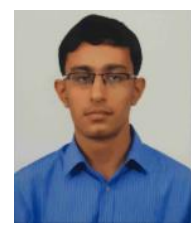

Mayank Pratap Singh, C.S.E, B.TECH,

SRM Institute of Science and Technology,

Chennai,Email:mayankpratapssingh@gmail.com

Mob.No.:-9952084909

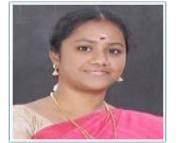

Mrs. M. Vaidhehi Rakesh, has completed her master of engineering in computer science engineering. Currently she is working as assistant professor in SRM IST Kattankulathur. She is having 7 years of teaching experience. Her research includes machine learning deep learning AL. Email: vaidhehi.m@ktr.srmuniv.ac.in 